\title{
Surgical Treatment of Leg Length Discrepancy and Knee Flexion Contracture Associated with a Congenital Absence of Quadriceps
}

\author{
Elad Holzer ${ }^{a} \quad$ Philippe Moisan $^{a} \quad$ Doron Keshet $^{\mathrm{a}}{ }^{\mathrm{b}} \quad$ Mitchell Bernstein $^{\mathrm{a}}{ }^{\mathrm{b}}$ \\ ${ }^{a}$ Faculty of Medicine, McGill University, Montreal, QC, Canada; ${ }^{b}$ Shriners Hospital for \\ Children, Montreal, QC, Canada
}

\section{Keywords}

Growth disturbances/limb length inequalities - Ilizarov - Taylor Spatial Frame · Absence of quadriceps $\cdot$ Knee contracture

\begin{abstract}
We report the case of an 18-year-old male with congenital absence of quadriceps and hypoplasia of the patella who presented with a significant leg length discrepancy (LLD) and knee flexion contracture. Surgical management was aimed toward lengthening the limb, stabilizing the joint, and correcting the knee flexion contracture. Correction of a significant congenital LLD and knee flexion contracture poses challenges due to long-standing altered biomechanics. These are rare conditions for which no accepted surgical algorithms exist. It is essential to anticipate the biomechanical consequences of limb lengthening and flexion contracture correction that might arise and plan comprehensive interventions accordingly.
\end{abstract}

(C) 2021 The Author(s).

Published by S. Karger AG, Basel

\section{Introduction}

Quadriceps femoris muscle is essential for stable knee extension, fluid ambulation, and optimal quality of life. Congenital aplasia of the quadriceps muscle is a rare condition mentioned only a handful of times in the orthopedic literature. Most case reports describe an

Elad Holzer and Philippe Moisan contributed equally to this work. 
association with ipsilateral aplasia or dysplasia of the patella [1-3]. Ideally, treatment and surgical correction should start before adulthood. Considering the rarity of such cases and absence of clear guidelines, we present our experience and core principles that guided our surgical management in a patient with this condition associated with limb length discrepancy (LLD) and flexion contracture of the knee.

\section{Case Report}

We present the case of an 18-year-old male treated for the following conditions: significant LLD (right shorter than left), congenital hypoplasia of the right quadriceps, right patellar hypoplasia, and right knee flexion contracture. The patient complained of limited mobility, severe limp, reduced athletic abilities, and recurrent falls. The patient ambulated with a $3.5-\mathrm{cm}$ external right shoe lift.

The patient had been examined carefully by our multidisciplinary team on various occasions throughout his childhood. The team includes pediatric orthopedic surgeons, medical geneticists, pediatricians, anesthesiologists, and a variety of other fellowship-trained physicians working at a specialized orthopedic hospital. The patient has never been diagnosed with any cardiac, pulmonary, or other malformation or dysmorphism. Taking into account the lack of comorbidities, the absence of family history, the unilateral nature of the patient's condition, and the current scientific literature, it was decided by the team that additional tests were not indicated.

On physical examination, the patient had an equinus short leg gait and a varus thrust of the right knee. Range of motion in the right knee lacked $20-25^{\circ}$ of extension with a flexion of $150^{\circ}$. There was no knee instability in the coronal or sagittal plane. The right quadriceps femoris had no trace of function with no active extension. Hamstring function was normal. The left knee had good range of motion $\left(0-150^{\circ}\right)$ with no signs of instability. No gross abnormalities were documented in either hip.

Standing hip to ankle radiographs demonstrated a significant LLD, with the right lower limb $82 \mathrm{~mm}$ shorter than the left lower limb (delta femur $58 \mathrm{~mm}$, delta tibia $24 \mathrm{~mm}$ ). Since the patient had a knee flexion contracture, lateral imaging was utilized to measure the LLD. On the lateral view of the right lower limb in maximal extension, knee flexion contracture combined with a hypoplastic patella and patellar alta was documented (Fig. 1a, b). The mechanical axis of the right lower limb passed through the middle of the knee joint with no apparent coronal malalignment. Measuring joint orientation line showed an oblique joint line with mLDFA $81^{\circ}$ and MPTA $81^{\circ}$.

MRI was performed at the age of 14 years to investigate the status of the anterior and posterior compartments of the left thigh. Findings were atrophy and hypoplasia of quadriceps muscle; the muscles of the posterior compartment were shown to be normal. The patella was proximally located and dysplastic. The distal femur was dysplastic with a shallow anterior femoral groove. Unfortunately, the MRI did not include knee structures. Therefore, the preoperative cruciate ligament status cannot be commented on.

Previous treatment included nonsurgical modalities such as physiotherapy, night-time bracing, and botulinum toxin injections to hamstrings. These conservative measures may have reduced the degree of knee flexion contracture with the unopposed knee flexor activity.

Surgical interventions were discussed at multiple multidisciplinary rounds throughout the patient's childhood. Our experienced team of fellowship-trained pediatric orthopedic surgeons considered various surgical options including Judet quadricepsplasty, femoral shortening, and patellar advancement knee flexors to extensors transfer. Considering the rarity of the patient's condition, the limited literature available, the unpredictability of the final results,

\section{Karger'}




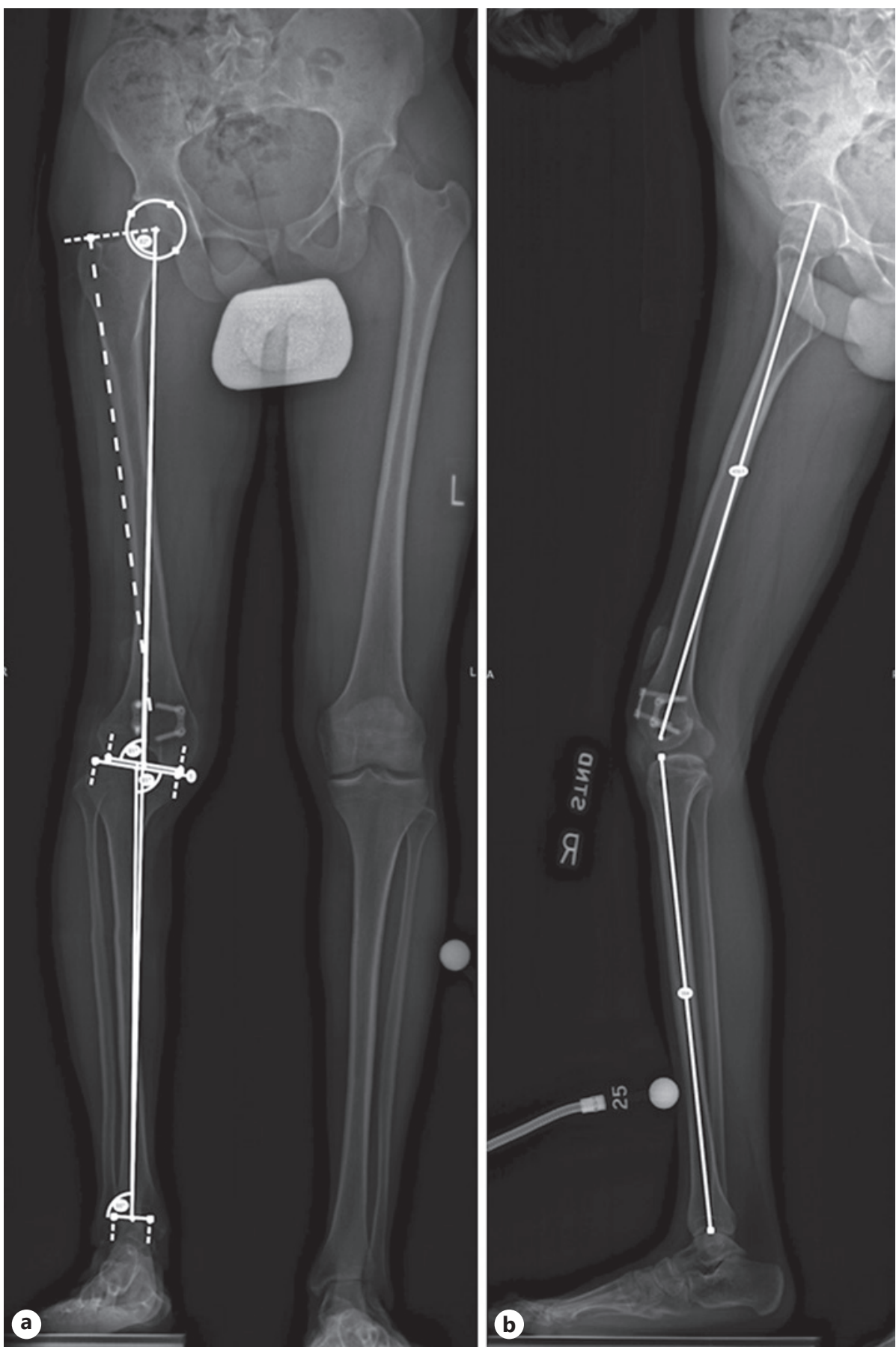

Fig. 1. a Anterior view computed radiography showing the initial limb length discrepancy. b Lateral view computed radiography of the right lower limb in maximal extension demonstrating the knee flexion contracture, the hypoplastic patella, and patellar alta.

and the bioethical principle of primum non nocere, together with the patient's family, it was determined that it would be best for surgical treatment to be delayed. Anterior distal right femur epiphysiodesis was eventually performed at age 14 with only minor improvement in the flexion contracture. Ultimately, the patient and his family, together with the surgical team, decided to pursue a definitive surgical management. 


\section{Case Reports in Orthopedic Research}

\begin{tabular}{l|l}
\hline Case Rep Orthop Res 2021:4:270-277 \\
\hline DOI: 10.1159/000521428 & $\begin{array}{l}\text { ○ 2021 The Author(s). Published by S. Karger AG, Basel } \\
\text { www.karger.com/cio }\end{array}$ \\
\hline
\end{tabular}

Holzer et al.: Congenital Absence of Quadriceps and Knee Contracture

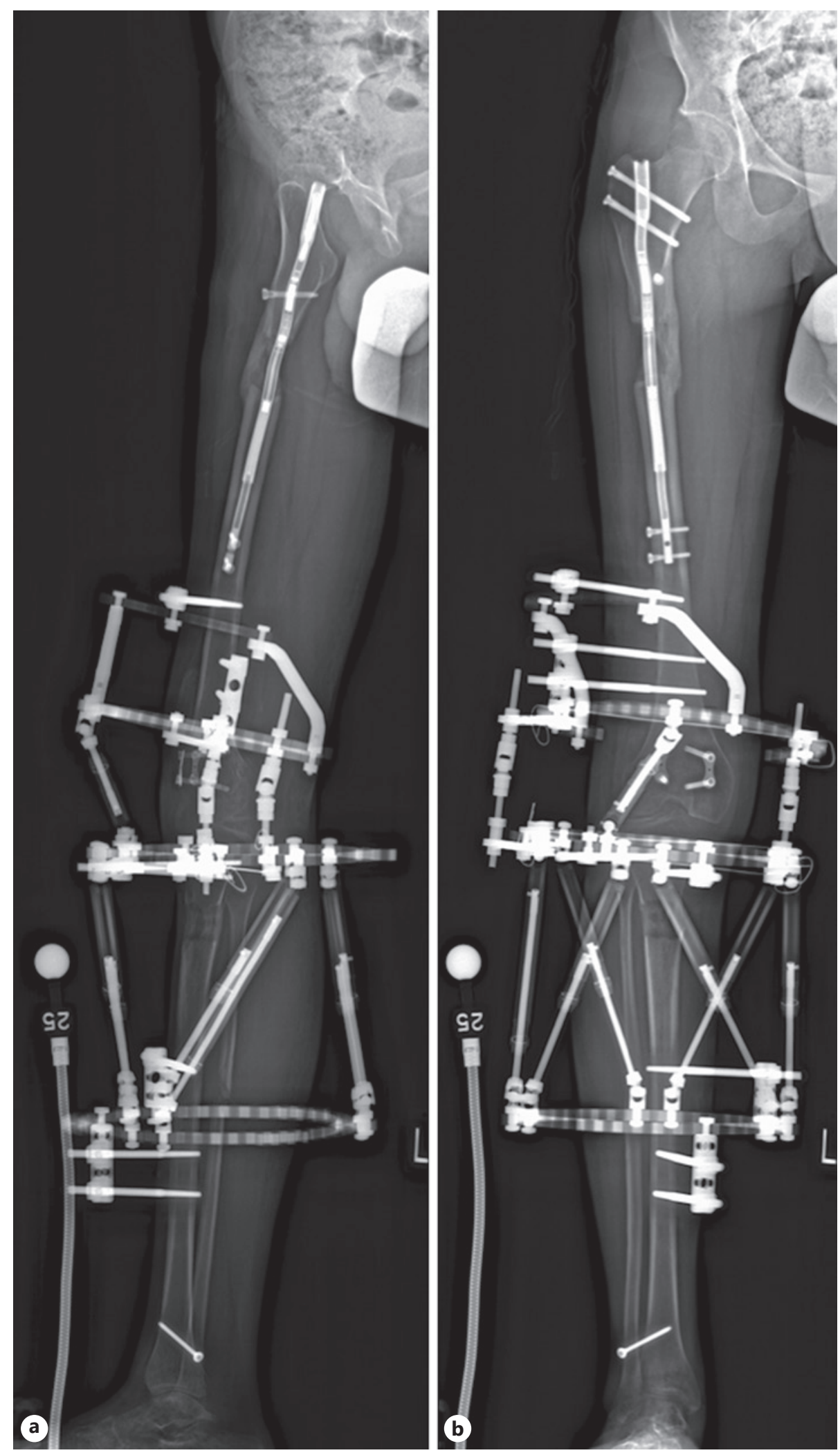

Fig. 2. a Postoperative computed radiography of the right leg in lateral showing the PRECICE anterograde nail, Taylor Spatial Frame, and hinge Ilizarov frame. b Postoperative computed radiography of the right leg in anterior view showing the PRECICE anterograde nail, Taylor Spatial Frame, and hinge Ilizarov frame. 
Case Reports in Orthopedic Research

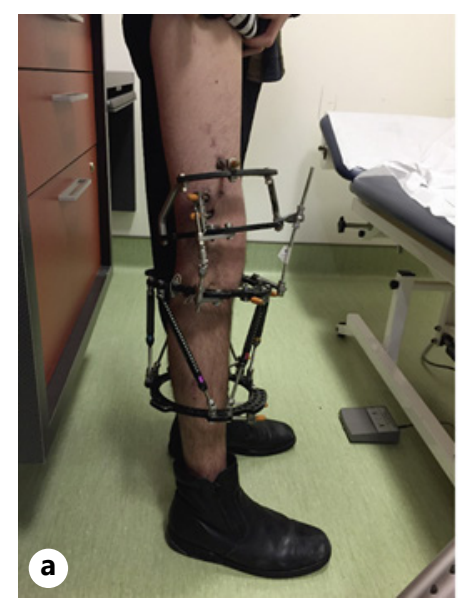

Fig. 3. a Lateral view image after 8 weeks of lengthening and contracture correction showing reduced limb length discrepancy and improved extension of the right knee. b Anterior view image after 8 weeks of lengthening and contracture correction showing reduced limb length discrepancy and improved extension of the right knee. c Posterior view image after 8 weeks of lengthening and contracture correction showing reduced limb length discrepancy and improved extension of the right knee.

Our treatment goals were to establish near-equal limb lengths with a knee that was fully extended. With equal limb lengths, the knee would be able to accommodate an extension knee brace that would maintain the extension and thus optimize ambulation. Due to the risk of subluxation/dislocation of the knee within the lengthening process, the knee had to be spanned for protection. Correction of the knee flexion contracture was performed concurrently by adding Ilizarov universal hinges.

The procedures performed during surgery were femoral intramedullary lengthening using PRECICE (Nuvasive Specialized Orthopaedics, Aliso Viejo, CA, USA) antegrade nail and proximal tibia lengthening using Taylor Spatial Frame (TSF) (Smith and Nephew, Memphis, TN, USA) which was used also to accommodate for a hinge Ilizarov frame over the knee for protection of the knee and correction of the knee flexion contracture (Fig. 2a, b). Gradual lengthening of the femur and tibia as well as gradual extension of the knee was carried out over a period of 8 weeks Figure 2 .

Re-intervention was necessary at 4 months after the surgery due to insufficient regenerate at the femoral lengthening site. This required autologous iliac crest bone graft injections (bone marrow aspirate concentrate injection) and knee extension contracture that required knee manipulation under anesthesia.

The TSF and the intramedullary nail were removed 7 months and 9 months postoperatively, respectively. In his latest clinical follow-up, 14 months status after TSF removal, the patient reported no pain. The examination showed full extension of his knee and $70^{\circ}$ of flexion, without any signs of knee instability and a normal neurovascular exam (Fig. 3a-c). Limb alignment radiographs showed 8-mm limb length discrepancy with the right side shorter and a valgus alignment of his right lower limb (MPTA 88, LDFA 84) (Fig. 4a, b).

\section{Discussion/Conclusion}

Confronted with a rare condition where consensus on treatment does not exist, we established a comprehensive approach and anticipated potential problems. Lengthening of the femur and the tibia without compromising knee stability and congruency was achieved by 


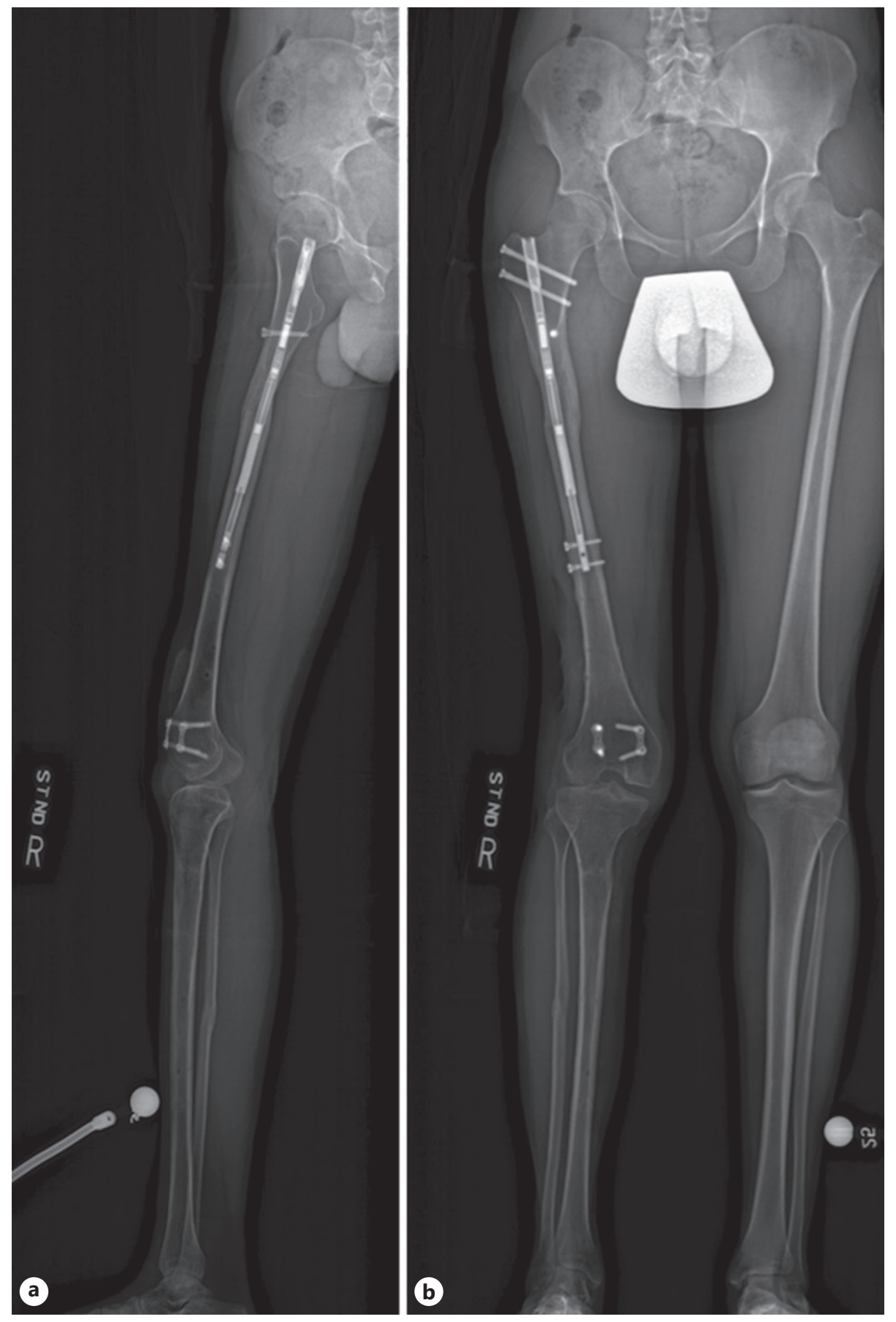

Fig. 4. a Lateral film postsurgical corrections computed radiography showing a residual 8-mm limb length discrepancy of the right side shorter and a valgus alignment of his right lower limb (MPTA 88, LDFA 84). b Anterior postsurgical corrections computed radiography showing a residual 8-mm limb length discrepancy of the right side shorter and a valgus alignment of his right lower limb (MPTA 88, LDFA 84).

spanning the knee with an Ilizarov hinge fixator which was also used to gradually correct the flexion contracture. Lengthening the tibia with a TSF was performed to accommodate for the Ilizarov hinge over the knee. 
Correction of the severe LLD and knee flexion contracture enabled the patient to have a much more efficient gait, eliminated the use of a shoe lift, and reduced complaints associated with an unstable knee. The patient was unable to retain the full knee flexion he had prior to treatment. We believe that his limited knee flexion at the end of lengthening $\left(70^{\circ}\right)$ is mainly the consequence of lack of soft tissue adaptation to lengthening.

As a result of the intervention, the patient's knee has become "brace-able." The patient wears an extension brace in order to maintain knee extension and to prevent a recurrence of the knee flexion contracture. He has an extension moment to the brace but can flex. Gaining full extension at the cost of losing some knee flexion has improved the patient's ambulatory abilities and overall well-being.

Lengthening of the femur was done with an antegrade intramedullary with expected lateralization of the mechanical axis. Tibial lengthening was performed using gradual correction with TSF and simultaneous correction of proximal tibia varus deformity. This added to the overall valgus alignment of the right lower limb. This valgus deformity could have been avoided by performing a distal femur osteotomy for coronal angulation correction and simultaneously lengthening the femur with a retrograde lengthening nail. Retrograde lengthening was avoided due to the altered biomechanics around the knee. The TSF was used to maintain a reduced knee joint during the lengthening, to lengthen the tibia, and to correct tibial malalignment (not limb malalignment). The plan was to stage the distal femur valgus procedure and to perform a second surgery with a distal femur opening wedge osteotomy. The second surgery will be performed in the near future.

The fact that joint narrowing of the hip joint may occur due to increased pressure during lengthening was considered. In our patient, the preoperative and postoperative images were reviewed, and no difference with regard to joint space was found.

Overall, correction of LLD and knee contracture poses additional challenges in congenital cases compared to correction in acquired ones. It is essential to anticipate problems that may occur when modifying the biomechanics of a patient. In this patient, anticipatory planning prevented potential issues from arising, although retrospectively, we may not have fully appreciated the extension contracture that occurred. After complete healing and extensive physical therapy, our patient is happier with ambulation, noticed significant quality of life improvement, and now attends university. If we were to encounter a patient with the same condition in the future, we would use the same principles and the same surgical techniques.

\section{Statement of Ethics}

This article was exempted from ethics review by the McGill Institutional Review Board on July 24, 2019. Written consent has been obtained from the patient to publish details of the medical case and any accompanying image.

\section{Conflict of Interest Statement}

The authors declare no conflicts of interest in this study.

\section{Funding Sources}

No funding was obtained for this research project or for any research relevant to the elaboration of this project.

\section{Karger'}


Case Reports

in Orthopedic

Research
Case Rep Orthop Res 2021;4:270-277

\begin{tabular}{l|l}
\hline DOI: $10.1159 / 000521428$ & ๑ 2021 The Author(s). Published by S. Karger AG, Basel
\end{tabular} www.karger.com/cio

Holzer et al.: Congenital Absence of Quadriceps and Knee Contracture

\section{Author Contributions}

Elad Holzer, Philippe Moisan, Doron Keshet, and Mitchell Bernstein have contributed to the chart review, redaction, and revision of this manuscript.

\section{Data Availability Statement}

The data that support the findings of this study are not publicly available due to ethical restrictions. Queries regarding the data in this article should be addressed to the corresponding author (P.M.).

\section{References}

1 Oner M, Halici M, Guney A. Congenital total absence of the quadriceps muscle and patella: a case report with computed tomography scan and three-dimensional reconstructions. J Pediatr Orthop B. 2013;22:322-4.

2 Shah AP, Vaidya S, Phadke S, Agashe M. Congenital aplasia of the patella and the distal third of the quadriceps mechanism: a case report. JBJS Case Connect. 2018;8:e79.

3 Varghese RA, Joseph B. Congenital aplasia of the patella and the distal third of the quadriceps mechanism. J Pediatr Orthop B. 2007;16:323-6. 